Pbilosophia Philosophia Scientiæ

Scientie Travaux d'histoire et de philosophie des sciences

CS 7 | 2007

Louis Rougier : vie et œuvre d'un philosophe engagé

\title{
Vies parallèles : Rougier et Cavaillès
}

\section{Pascal Engel}

\section{OpenEdition}

\section{Journals}

Édition électronique

URL : http://journals.openedition.org/philosophiascientiae/428

DOI : 10.4000/philosophiascientiae.428

ISSN : 1775-4283

Éditeur

Éditions Kimé

Édition imprimée

Date de publication : 1 janvier 2007

Pagination : $19-48$

ISBN : 978-2-84174-412-1

ISSN : $1281-2463$

Référence électronique

Pascal Engel, «Vies parallèles : Rougier et Cavaillès », Philosophia Scientiæ [En ligne], CS 7 | 2007, mis en ligne le 08 juin 2011, consulté le 16 janvier 2021. URL : http://journals.openedition.org/ philosophiascientiae/428; DOI : https://doi.org/10.4000/philosophiascientiae.428 


\title{
Vies parallèles : Rougier et Cavaillès
}

\author{
Pascal Engel \\ Université de Genève
}

«Je souhaite que la légende, épurée par la raison, se soumette à elle, épurée par l'histoire » Plutarque, Vies Parallèles

\section{Introduction}

Il y a quelque chose d'incongru, et même de scandaleux et d'indécent, à accoler, ne serait-ce que pour les opposer, le nom de Jean Cavaillès à celui de Louis Rougier. Le premier est un héros de la résistance, le second est surtout connu pour ses compromissions avec le régime de Vichy. Cette circonstance, et les engagements politiques ultérieurs de Rougier les rendent absolument antithétiques. Je voudrais être absolument clair d'entrée de jeu : il n'est pas question pour moi ici de réhabiliter en quoi que ce soit la figure de Rougier ni la partie de son œuvre et de ses activités qui offrent à présent surtout un intérêt pour les historiens. La question qui m'intéresse à son sujet est plutôt la suivante : comment se fait-il qu'un philosophe de grand talent, pionnier dans son pays dans bien 
des domaines, de la philosophie des sciences à la promotion du libéralisme, ait pu se retrouver l'un des pères de la Nouvelle Droite et associé à l'extrême droite française au point de devenir une sorte de paria? La question dépasse le cas spécifique de Rougier. C'est précisément parce que Cavaillès n'a pas suivi ce chemin que le contraste m'intéresse.

La considération parallèle des trajectoires de Cavaillès et de Rougier invite à s'interroger sur les liens, ou l'absence de liens, que peuvent avoir les poursuites intellectuelles et les poursuites politiques. Considérez en effet les cas suivants, décrits de manière volontairement caricaturale :

(1) Le plus grand philosophe et logicien de l'antiquité se prononce pour l'esclavage.

(2) Le plus grand logicien depuis Aristote exprime dans son journal des opinions violemment antisémites.

(3) Un philosophe considéré par certains comme le plus grand du $\mathrm{XX}^{\mathrm{e}}$ siècle entre au parti nazi.

(4) Le représentant le plus ardent de l'existentialisme engagé attend tranquillement que la guerre se passe.

(5) Le philosophe des mathématiques le plus brillant de sa génération s'engage dans la résistance et y meurt en héros.

(6) L'unique représentant français du positivisme logique s'engage auprès de Vichy et devient l'un des inspirateurs de l'extrême droite.

On pourrait prendre des dizaines d'exemples chez les intellectuels ou les écrivains, mais ma question ici porte sur les philosophes. Un philosophe n'est pas n'importe quel type d'intellectuel. Il est supposé avoir, dans sa vie et sa pensée, une unité qu'on n'attend pas nécessairement chez un écrivain, un journaliste ou un artiste, et ce qu'il écrit est supposé répondre à un certain nombre d'exigences quant à la recherche du vrai et quant à certaines valeurs intellectuelles et morales. Comme le dit Louis Rougier lui-même : «Un philosophe est un homme qui a une hiérarchie de valeurs dans la tête et qui n'est pas prêt à sacrifier le nécessaire au contingent, le définitif au provisoire, l'éthique à la politique. » [Rougier 1945b, 276] Voilà de belles paroles. Toute la question est dans le fait que les idées ne s'accordent pas toujours avec les actes. Les opinions sont très diverses également sur les relations entre les deux. Il semble y avoir trois thèses possibles :

(1) La thèse de l'Implication stricte : il y a un lien nécessaire entre l'engagement théorique d'un philosophe et son engagement politique et vice versa.

(2) La thèse de l' Implication matérielle : il n'y a qu'un lien contingent entre les deux, et ils ne s'impliquent pas mutuellement, voire pas de lien du tout. 
(3) La thèse de la fonction partielle : il y a des recoupements partiels ou des recoupements, mais aucun lien intrinsèque.

La thèse (1) de l'implication stricte est défendue par Ryle, qui soutenait, au sujet de Heidegger, qu'une crapule ne peut pas être un bon philosophe et vice versa, et par Althusser, qui soutenait qu'un philosophe fait nécessairement de la «lutte de classes dans la théorie ». Benedetto Croce semble défendre la thèse (2), celle de l'implication seulement matérielle quand il dit à propos, toujours à propos de Heidegger :

« Je viens de lire la totalité du discours ( du Rectorat) de Heidegger, qui est à la fois stupide et servile. Je ne suis pas surpris du succès que sa manière de philosopher aura sans doute pendant un temps - ce qui est vide et général a toujours du succès. Mais cela ne produit rien. Je pense aussi que cela n'aura aucun effet sur la politique, mais il déshonore la philosophie, et c'est une pitié aussi pour la politique, en particulier la politique du futur. $»^{1}$

Il faut distinguer plusieurs choses :

(a) la relation entre les engagements théoriques et les engagements pratiques d'un philosophe;

(b) la relation entre ses thèses théoriques et ses thèses au sujet de la pratique;

(c) la question générale des relations entre valeurs théoriques et cognitives d'une part et valeurs pratiques et éthiques de l'autre.

Dans ce qui suit, je ne m'intéresserai qu'à (a) et (b), et je laisserai de côté la question philosophique (c), mais on peut espérer que le parallèle Rougier / Cavaillès puisse l'éclairer indirectement. ${ }^{2}$

\footnotetext{
${ }^{1}$ Cité par Simon Blackburn, « Enquivering », CR de M. Heidegger, Contributions to Philosophy, The New Republic, 30 oct. 2000.

${ }^{2}$ Antonia Soulez m'a fait remarquer qu'il manque à la taxinomie ci-dessus la catégorie suivante : (b') le lien entre les thèses sur la pratique et l'engagement pratique. J'en dis ici assez peu. Cavaillès ne s'est pas exprimé sur les premières, alors que Rougier l'a beaucoup fait dans ses écrits politiques. La question de savoir si le libéralisme économique et politique de Rougier a des liens avec son engagement pétainiste et d'extrême droite est évidemment au cœur du jugement que les contemporains ont porté sur lui. Mais là aussi il ne semble pas y avoir de lien nécessaire. Le parallèle qui suit sera naturellement, puisqu'il appartient à un ensemble d'essais consacrés à Rougier, disproportionné au bénéfice de Rougier. On aura, je l'espère, compris, qu'il ne s'agit pas pour moi d'être aussi détaillé sur l'oeuvre de Cavaillès, à présent bien connue, que sur celle de Rougier. Cavaillès sera ici utilisé en contrepoint, pour éclairer, par antithèse, Rougier.
} 


\section{Vie de Cavaillès}

La vie de Cavaillès n'est pas très difficile à raconter, et elle est plus que connue, presque légendaire. Elle pourrait presque se raconter dans le style des brèves de Félix Fénéon : « Il fit des mathématiques et de la résistance, la seconde lui fut fatale ». Né en 1903 à St Maixent où son père était officier, il fut éduqué dans la religion protestante. Il fait ses études à Louis le Grand et entre cacique à l'Ecole normale Supérieure en 1923. Il étudie la philosophie à la Sorbonne et fait une licence de mathématiques, et sous l'influence de Brunschvicg s'intéresse à la philosophie des mathématiques. Il fait son service militaire comme élève officier de réserve à Saint Cyr en 1927 et obtient l'agrégation la même année. En 1929, puis en 1930-31, il fait des séjours d'études en Allemagne avec une bourse Rockefeller, et est agrégé répétiteur à l'Ecole normale supérieure (où il assiste notamment à des cours de mathématiques dispensés par Jacques Herbrand aux conscrits). Il assiste au débat Heidegger / Cassirer à Davos, et écrit des articles sur la crise du protestantisme allemand, ${ }^{3}$ participe au Congrès de philosophie de Prague en 1934, à celui de Paris en 1935. De 1936 à 1938 il est professeur au lycée d'Amiens. En 1938 il passe sa thèse de doctorat es Lettres, Méthode axiomatique et formalisme, et sa thèse complémentaire, Remarques sur la formation de la théorie abstraite des ensembles (Cavaillès 1938a,b). Il obtient alors un poste de maître de conférences à l'université de Strasbourg. En 1939, il est mobilisé, en 1940 fait prisonnier. Il s'évade et rejoint son poste à Strasbourg, au moment où cette université est repliée à Clermont Ferrand. Là il commence, dès 1940, son activité dans la résistance, et fonde le mouvement «Libération ». En 1941, il est chargé de cours de logique à la Sorbonne. Il fonde le réseau «Cohors » qui couvre la résistance sur presque toute la partie nord de la France. Arrêté en 1942 par la police de Vichy, profite des loisirs de la prison pour écrire Sur la logique et la théorie de la science, et s'évade. Révoqué par Vichy, il fait plusieurs voyages à Londres. Il est arrêté le 28 août 1943 à Paris. Torturé, incarcéré à Fresnes puis à Compiègne, il sera transféré à la Citadelle d'Arras et fusillé le 17 février 1944. Enterré à Arras sous une croix de bois portant la mention "inconnu ${ }^{\circ} 5$ ". Compagnon de la libération.

Cavaillès a laissé une œuvre brève, souvent elliptique, inachevée, consistant essentiellement en ses deux thèses, la courte monographie

\footnotetext{
${ }^{3}$ Ces articles (publiés dans [Heinzmann (éd.) 1998]) manifestent une maturité politique très précoce, et une grande lucidité sur le nazisme montant en Allemagne. On ne peut pas dire que Cavaillès ne se soit pas engagé contre les nazis plus tard sans le faire en toute connaissance de cause. Voir [Cavaillès 1932a, 1932b, 1933, 1934a $1934 \mathrm{~b}]$.
} 
Sur la logique et quelques articles (Cavaillès 1994). Ses écrits portent essentiellement sur l'histoire des mathématiques et en partie sur leur philosophie et sur celle de la connaissance et de la logique. Ils ont été fort célébrés après la guerre mais on a surtout l'impression, à lire les premiers commentaires, qu'on y loue surtout l'alliance de la philosophie, des mathématiques et de l'héroïsme du résistant, sans comprendre beaucoup la portée de ses vues ni sans connaître très bien ses références. ${ }^{4}$ Le premier à avoir commenté les conceptions de Cavaillès en philosophie fut son élève Gilles Gaston Granger dans son article « Cavaillès ou la montée vers Spinoza »[Granger 1947], très pénétrant mais bref. Ce n'est que plus tard que sont venus des commentaires et études spécifiques [Desanti 1968], [Sinaceur 1994], [Heinzmann 1987], [Heinzmann 1989] et [Cassou-Noguès 2001].

Il n'est pas facile de caractériser la philosophie des mathématiques de Cavaillès en termes des divisions familières (platonisme, constructivisme, formalisme, logicisme, voire nominalisme), à la différence de Lautman, par exemple, qui prend parti clairement pour le platonisme. Le langage de Cavaillès est souvent ambigu. Ainsi il se réclame de l'expérience mathématique, mais ce qu'il entend par là est aux antipodes d'une conception empiriste comme celle qu'a pu défendre Stuart Mill, ou de nos jours Philip Kitcher [Kitcher 1981] :

«Par expérience, j'entends un système de gestes, gouverné par une règle, et soumis à des conditions indépendantes de ces gestes. » [Cavaillès \& Lautman 1939 (1994), 601]

Cavaillès ne dit pas ce que sont ces « gestes » ni en quoi consistent les 《règles » qui les gouvernent. On pourrait comprendre par là quelque chose comme une expérience subjective, ou une forme de genèse du type de celle qu'envisage Piaget quand il parle de la genèse des opérations logiques et mathématiques élémentaires. Mais ce n'est évidemment pas en ce sens qu'il entend s'exprimer, lui qui rejette très clairement, au début de Sur la logique, toute forme de psychologisme en logique et en mathématiques. La notion de 《 geste » a conduit certains interprètes à y voir une théorie des objets mathématiques enracinée dans une phénoménologie du vécu mathématique interprétée comme une genèse dans le corps, à la manière de Merleau-Ponty [Cassou-Noguès 2001]. En fait Cavaillès semble entendre par «expérience» non pas une propriété personnelle du sujet connaissant, mais l'expérience historique, collective des mathé-

\footnotetext{
${ }^{4}$ Cavaillès fut l'un des premiers philosophes français à avoir lu attentivement Bolzano et Wittgenstein notamment. A l'époque aussi où ses collègues français s'intéressaient surtout à Heidegger, il lisait attentivement Transzendentale und Formale Logik de Husserl.
} 
maticiens au cours de l'histoire de cette discipline. Il nous dit que par «empirisme » et « expérience » il entend :

« la simultanéité de la mathématique avec son travail présent, empirisme encore puisqu'il ne s'agit que de décrire le travail effectif, mais empirisme de la pensée en acte, sans autre référence que le devenir imprévisible des mathématiques » [Cavaillès 1938a, 18-26]

Mais c'est certainement un sens bien spécial du terme «empirisme» qui est ainsi visé. On trouve une ambiguïté semblable dans la notion d'intuition. Cavaillès est très clair sur le fait qu'il n'entend pas par là l'intuition au sens kantien, ni husserlien, à laquelle il reproche d'être statique ou « un arrêt sans pensée » [Cavaillès 1941]. Il faut comprendre que l'intuition est un progrès constant. Mais qu'est-ce qu'une intuition dynamique ou qui progresse? On ne le sait pas.

Cavaillès est un élève de Léon Brunschvicg. Ce dernier soutenait que la conscience - rationnelle - humaine progresse à travers l'histoire, s'incarnant dans les mathématiques (mais pas dans la logique, qu'à l'instar de toute la tradition française il considérait comme stérile, ce qui le conduisait à rejeter avec dédain 《la logistique» de Russell). Il reste manifestement quelque chose de cette conception historique de la raison chez Cavaillès, même si ce dernier ne souscrit pas à l'idéalisme de son maître. En particulier, comme on vient de le voir, Cavaillès soutient que le devenir mathématique n'est réglé par aucun plan ni telos préétabli, et qu'il est «imprévisible ». Imprévisible, mais en même temps objet d'une «nécessité génératrice », qui, comme le disent les fameuses dernières lignes de Sur la logique et la théorie de la science, est également « dialectique » :

« Ce n'est pas une philosophie de la conscience mais une philosophie du concept qui peut donner une doctrine de la science. La nécessité génératrice n'est pas celle d'une activité, mais d'une dialectique. » [Cavaillès 1947, 560]

On a compris souvent par là que Cavaillès voulait affirmer le primat de l'objectivité mathématique sur les actes de la conscience. On a compris aussi qu'il entendait parler de la nécessité mathématique en sens spinoziste, contre une conception de type kantien ou husserlien. Ou encore on y a vu une anticipation du structuralisme mathématique au sens de Bourbaki. Le terme de «dialectique »a excité l'esprit des marxistes et des hegéliens. D'autres au contraire ont soutenu que Cavaillès ne voulait pas du tout déloger la conscience de son primat, et fondait plutôt une philosophie phénoménologique. Gilles Granger qui fut son élève à la 
Sorbonne, a donné, à mon sens, l'interprétation la plus intéressante de la distinction, empruntée elle-même par Cavaillès à Husserl, entre paradigme et thématisation : la pensée mathématique crée dans un premier temps des formes en construisant des procédure formelles, vidées de leur contenu, puis, dans un second temps, crée des structures portant sur ces procédures, qui deviennent des «thèmes », et il y a ainsi des degrés du «contenu formel »(Granger 1988, 1994). Granger a rapproché la notion chez Cavaillès de «travail mathématique» à celle de développement d'une essence singulière, mais cette lecture doit autant à la propre philosophie des mathématiques de Gilles Granger qu'à Cavaillès lui-même. ${ }^{5}$

Il semble très difficile de tirer de ces suggestions quelque chose comme une philosophie des mathématiques, du moins si l'on entend par là une réponse articulée et explicite à des questions telles que : y-t-il des vérités en mathématiques? Si oui, les vérités mathématiques sont-elles $a$ priori? analytiques ou synthétiques? Sur quelles sorte d'entités portentelles? La notion de démonstration est-elle première par rapport à celle de vérité? etc. Certes Cavaillès ne cesse de discuter de ces questions, mais sa réponse est très difficile à saisir. Qu'est-ce une vérité mathématique qui est à la fois objet d'intuition et expérience, nécessaire mais imprévisible, historique mais non contingente? Qu'est-ce exactement qu'être spinoziste en philosophie des mathématiques? Qu'est-ce que cette synthèse entre l'intuition et l'axiomatique, entre le logique et le non logique que Cavaillès a tenté de faire? Cavaillès a surtout laissé des travaux d'histoire des mathématiques, et il défend certainement, à l'instar de Brunschvicg, ce que l'on a appelé une épistémologie historique des mathématiques, dans laquelle l'histoire des concepts et des théories est essentielle. Mais il y a histoire et histoire. Cavaillès anticipe-t-il la philosophie silencieuse au sens de Desanti, selon laquelle le philosophe des mathématiques doit se placer à l'intérieur des travaux du mathématicien, par une sorte de paraphrase historique subtile, et surtout ne pas dire quoi que ce soit qui relèvera d'une philosophie des mathématiques? Il s'oppose clairement au logicisme, en particulier viennois, et refuse une réduction du mathématique au logique, et il refuse tout autant le constructivisme et l'intuitionnisme. Il a certainement voulu montrer qu'il n'y avait de philosophie des mathématiques qu'en acte, au plus près des pensées des mathématiciens et de leur histoire, et cela suffit certainement à distinguer son approche de bien d'autres. Mais, au delà, il faut reconstruire sa position, et toute interprétation de son œuvre court le risque de la surinterprétation. Le caractère elliptique des remarques de Cavaillès est-il voulu ou bien est-il

\footnotetext{
${ }^{5}$ Voir notamment [Granger 1996] cité d'après [Heinzmann (éd.) 1998].
} 
dû simplement au fait qu'il n'a pas eu le temps d'en dire plus? Je penche pour la seconde réponse, mais il est certain que la clarté de son action n'a pas pour pendant celle de ses vues sur les mathématiques. Il faut un travail complexe d'exégèse et de reconstruction pour accéder à celles-ci. ${ }^{6}$

Quoi qu'il en soit, quel peut être le lien entre cette œuvre théorique et l'engagement de son auteur dans la Résistance? On a beaucoup écrit et spéculé là-dessus, en suggérant notamment, à partir d'une remarque que Cavaillès fit dans une lettre adressée à Raymond Aron, ${ }^{7}$ que le lien entre les deux résidait dans un sens, tout spinoziste, de la nécessité. On a également soutenu que l'éducation protestante de Cavaillès l'avait particulièrement prédisposé à un tel engagement. Je reviendrai plus bas sur ce point, mais je tendrais à penser que c'est surtout l'expérience de la débâcle, chez un officier, lui-même fils d'officier, qui a d'abord été déterminante. Quand on lit L'étrange défaite de Marc Bloch [Bloch 1946], qui lui aussi était un universitaire de Strasbourg, officier durant la guerre de 14 et remobilisé en 1939-40, on peut, je crois, comprendre quelle était la mentalité de ces soldats qui n'acceptèrent pas la défaite et avaient un sens élevé de leur devoir. La réaction à l'occupant leur apparaissait comme une évidence et une nécessité qui ne se discute pas. Quand on voit les biographies de tant d'intellectuels sous l'occupation, cela apparaît comme exceptionnel. Mais cela n'a, quand on considère nombre de parcours de résistants, rien de vraiment exceptionnel. Certes, comme l'a dit à de nombreuses reprises Georges Canguilhem, il y a une harmonie évidente entre la conception que Cavaillès se faisait de la philosophie et son action de résistant, mais je ne vois pas pourquoi Cavaillès aurait eu un sens du devoir particulièrement en tant qu'intellectuel ou en tant que philosophe, car toutes sortes de gens qui n'avaient pas de culture universitaire et qui ne venaient pas du même milieu ont été capables d'avoir cette réaction. J'y reviendrai plus bas, mais il ne me semble pas y avoir grand-chose de plus à dire sur cette question que ce que dit Robert Paxton quand il dresse à la fin de son ouvrage sur Vichy le «bilan moral » de la période :

« Lorsqu'il fallu choisir - et le choix était dramatique - entre deux solutions : faire son travail, et courir des risques moraux et abstraits, ou pratiquer la désobéissance civile, donc s'exposer à des dangers physiques immédiats, la plupart des Français ont poursuivi leur travail. L'auteur et les lecteurs de cet ouvrage auraient peut-être été tentés, hélas, d'en faire

\footnotetext{
${ }^{6}$ La lecture de Hourya Sinaceur [Sinaceur 1994] est certainement la plus complète et la plus élaborée.

${ }^{7}$ Citée par [Canguilhem 1967 (1994), 674].
} 
autant. Il est parfois dans l'histoire d'un pays un moment cruel où pour sauver ce qui donne son vrai sens à la nation, on ne peut pas ne pas désobéir au gouvernement. En France, c'était après juin 1940.» [Paxton 1973 (1977), 438]

\section{$3 \quad$ Vie de Rougier}

La vie de Rougier est moins transparente que celle de Cavaillès, c'est le moins que l'on puisse dire, et le récit qui suit en sera nécessairement plus long. ${ }^{8}$ A la différence de ce dernier, il est né dans une famille catholique de la bourgeoisie lyonnaise; son père était médecin, son grand-père professeur d'économie politique à la Faculté de Lyon. Il fait ses études au lycée Ampère, puis à la faculté des lettres de Lyon, où il a comme professeur Edmont Goblot, qui incarnait alors la logique traditionnelle et avait peu de sympathie pour la «logistique » comme on l'appelait alors. Il passe l'agrégation de philosophie en 1914, la même année que Jean Nicod, mais rien n'indique qu'ils se soient connus. De constitution fragile, il est réformé. Il enseigne au lycée, et une bonne partie de sa carrière s'est faite à l'étranger, en partie par goût, mais sans doute également en partie parce qu'il n'est pas du sérail de la rue d'Ulm ni de la Sorbonne. Dès 1917, alors qu'il est professeur à Alger, il commence à publier des articles sur la logique et la philosophie des sciences, défendant la logique contemporaine à la suite de Couturat [Rougier 1917b], le conventionnalisme de Poincaré (son premier article s'appelle « la mort des vérités nécessaires »). L'après-guerre, durant laquelle il est professeur au lycée français de Rome deux ans (1920-22), est pour lui une période d'intense activité intellectuelle. En 1919 il publie un ouvrage sur la matérialisation de l'énergie, sur la théorie de la relativité et les quanta. En 1920 il passe sa thèse sur Poincaré, ${ }^{9}$ et sa thèse complémentaire sur les paralogismes $\mathrm{du}$ rationalisme. Il multiplie les introductions, travaux éditoriaux, ouvrages populaires et savants. Après avoir espéré, en vain, obtenir à Lyon la chaire de son ancien professeur, Goblot, il devient professeur à la faculté des Lettres de Besançon en 1924. ${ }^{10}$

\footnotetext{
${ }^{8}$ Je dois beaucoup à [Marion 2004] dans cette biographie. Mais comme on va le voir, je suis en désaccord sur certains points.

${ }^{9}$ Le directeur de sa thèse était Gaston Milhaud [Marion 2004], qui a joué un rôle non négligeable dans la promotion du conventionnalisme en philosophie des sciences ; cf. [Brenner 2003].

${ }^{10}$ Les relations entre Rougier et Goblot semblent ne pas avoir été très bonnes, notamment parce que ce dernier avait peu d'estime pour ce que l'on appelait la « logistique » dont Rougier s'était fait le défenseur ardent. Cf. les polémiques avec Goblot, in [Rougier 1916a et 1919b].
} 
Dès cette époque il ne cesse d'écrire en parallèle sur les questions de philosophie des sciences et sur celles de politique et de religion. Ses grands inspirateurs sont Taine, Renan et Poincaré, dans une certaine mesure Lévy-Bruhl. Rougier semble avoir lu la Prière sur l'Acropole dès sa plus tendre enfance et vibré aux paroles célèbres : «Ô noblesse! Ô beauté simple et vraie! Déesse dont le culte signifie raison et sagesse, toi dont le temple est une leçon éternelle de conscience et de sincérité, j'arrive tard au seuil de tes mystères; j'apporte à ton autel beaucoup de remords. Pour te trouver, il m'a fallu des recherches infinies. L'initiation que tu conférais à l'athénien naissant par un sourire, je l'ai conquise à force de réflexions, au prix de longs efforts. ». A Taine et à Renan, il emprunte le positivisme, à Poincaré le conventionnalisme, qu'il va radicaliser. A Lévy-Bruhl, héritier du positivisme comtien, il emprunte la notion de mentalité primitive, notamment en parlant de «mentalité scolastique ». Sa thèse complémentaire Les paralogismes du rationalisme contient en germe toutes les idées qu'il va développer jusqu'à la fin de sa carrière. Le rationalisme n'est pas seulement une théorie de la connaissance, mais aussi une métaphysique et une morale, celle de l'égalité. Dès ce livre Rougier prend l'habitude de faire des généralisations historiques audacieuses. En supposant l'existence d'une raison identique chez tous les hommes, le rationalisme est non seulement un produit des idéaux judéo-chrétiens, mais il est responsable de ceux de la Révolution française et de la démocratie contemporaine. Mais, nous dit Rougier, le rationalisme est basé sur des paralogismes, dont le principal consiste à transférer la nécessité formelle des déductions logiques et mathématiques aux principes euxmêmes sur lesquels ces déductions reposent : on crée ainsi le mythe de vérités nécessaires, a priori et analytiques, alors qu'on n'a affaire qu'à des principes synthétiques, variables, conventionnels.

Tout comme il radicalise les thèses de Poincaré en philosophie des sciences, il radicalise celles de Taine et de Renan, ainsi que de celles de Lévy-Bruhl. En 1925, il publie sa vaste somme de 800 pages, La scolastique et le thomisme. Elle est destinée à la fois à prolonger les analyses de sa thèse, en montrant que le thomisme commet les paralogismes déjà dénoncés, et à montrer qu'il est l'incarnation même du catholicisme. L'ouvrage était provocateur, à un moment où le néo-thomisme renforçait ses positions avec Maritain et Gilson. Il donna lieu à une polémique, plusieurs dominicains l'ayant accusé de plagiat. Mais Lalande, dans son compte rendu de la philosophie française paru dans la Philosophical Review (à une époque où la philosophie universitaire française intéressait encore les Américains) trouve que Rougier s'attaque à un moulin à vent [Lalande 1925]. 
Les écrits de Rougier sur la politique et la religion ne sont pas moins vigoureusement polémiques que ses écrits de philosophie des sciences. Dès les années 20, il se spécialise dans ce qui sera l'une des passions de son existence, la polémique anti-chrétienne. Il dirige aux Editions du vingtième siècle la collection Les maîtres de la pensée antichrétienne, où il fait paraître en 1926 ce qui sera l'un de ses livres les plus célèbres, Celse ou Le conflit de la civilisation antique et du christianisme primitif, suivi du Discours vrai de Celse. L'ouvrage sera souvent réédité, en particulier par Alain de Benoist et ses associés de la nouvelle droite. ${ }^{11}$

Dans Les paralogisme du rationalisme, Rougier associait déjà la croyance à la raison et à l'égalité des hommes entre eux à une forme de mystique. En 1929, il reprend systématiquement ce thème dans La mystique démocratique, dans lequel il dénonce d'affilée, et souvent en laissant entendre qu'il ne s'agit que d'une longue équation, le judaïsme, le christianisme, l'idéologie égalitariste de la Révolution française, le socialisme et le communisme et la démocratie comme participant d'une même mystique. Le livre est placé sous l'invocation de Pareto et Rougier ne fait pas mystère de son admiration pour sa théorie des élites. Il entend traquer dans l'égalitarisme démocratique les mêmes paralogismes et contradictions que ceux qu'il a dénoncés dans le rationalisme en théorie de la connaissance.

Les années 1930 sont une période d'intense activité pour Rougier et ses écrits et activités portent essentiellement sur des questions politiques et économiques. Il enseigne au Caire deux ans (1934-36). Il commence à cette époque ses contacts avec le Cercle de Vienne, surtout avec Schlick, dont il est un ami personnel et qu'il rencontre en Autriche, mais aussi avec Reichenbach, avec qui il a une correspondance abondante, ${ }^{12}$ ainsi qu'avec Neurath. Il organise le premier colloque de philosophie scientifique à la Sorbonne en 1935 puis un autre colloque de philosophie des sciences en 1937, apparaissant comme le principal représentant du Cercle de Vienne en France. Et surtout son activisme le place au cœur d'un groupe influent d'économistes, de politiques et d'intellectuels oeuvrant pour le renouveau du libéralisme; il est en 1938 l'organisateur du colloque Walter Lippmann à Paris, un épisode à présent bien connu et bien documenté [Denord 2002], [Dard 2002]. Durant la même période, il publie intensivement en philosophie sociale et économique : notamment

\footnotetext{
${ }^{11}$ [Rougier 1926a]. L'ouvrage est reparu chez Pauvert en 1965, puis, avec une préface de A. de Benoist, aux Editions Copernic en 1977 [Rougier 1977]. L'édition la plus récente est chez Phébus en 1999.

${ }^{12}$ La correspondance Rougier-Reichenbach se trouve aux Archives of Scientific Philosophy à l'Université de Pittsburgh.
} 
Les mystiques politiques (1935a), Les mystiques économiques (1938b), qui reprennent toutes le même schème que La mystique démocratique.

En 1939, Rougier a publié plus de 15 ouvrages, des dizaines d'articles. Est-il pour autant un philosophe influent et connu? Ses polémiques antiscolastiques ont eu un certain succès, mais lui ont surtout procuré des ennemis. Ses écrits de philosophie des sciences sont estimés, mais marginaux, et ils ne lui ont pas procuré la reconnaissance universitaire qu'il escomptait. Après avoir échoué à obtenir la chaire de Goblot à Lyon, et obtenu un poste à Besançon, il a surtout enseigné à l'étranger. Il est vrai qu'il n'était pas facile, dans la philosophie française de l'entre-deux guerres, de se consacrer à la logique et de défendre des thèses positivistes. Ni Couturat, ni Nicod, ni Herbrand n'avaient, durant la même période, rencontré beaucoup de succès en se posant en défenseurs de ce que l'on appelait la «logistique ». Les mathématiciens, comme Poincaré, Borel, Hadamard, ou Fréchet, puis plus tard Weil et Chevalley ne trouvaient aucun intérêt à la logique, ${ }^{13}$ et ils défendaient tous une forme d'intuitionnisme. La philosophie des sciences était dominée par la figure de Brunschvicg, qui considérait la logique comme stérile et le logicisme comme une absurdité. Rougier ne pouvait pas non plus avoir d'atomes crochus avec Meyerson, qui défendait une conception de la raison diamétralement opposée à la sienne. Ses ouvrages anti-chrétiens et anti-scolastiques lui avaient valu l'antipathie profonde des milieux catholiques à l'époque encore très influents dans l'université. Mais même des auteurs a priori favorables au type de philosophie des sciences qu'il représentait, comme André Lalande, jugeaient que pour lui «l'histoire était prétexte à établir des conclusions dogmatiques », et Alexandre Koyré, dans un long compte rendu qu'il consacre à La scolastique et le thomisme, n'a aucun mal à mettre en valeur les faiblesses du livre, qui englobe sous la mentalité scolastique, à peu près toute la philosophie hormis la science positive [Koyré 1926]. En critiquant aussi bien le kantisme, que l'idéalisme spiritualiste, l'intuitionnisme mathématique et la néo-scolastique, on peut dire que Rougier s'est mis à dos à peu près toutes les tendances de la philosophie française de l'époque. Il semble même qu'il prenne un malin plaisir à ce style «Etiam si omnes ego non » (qui est la devise de Benda). Seul, apparemment, Bergson n'est pas visé par ses écrits, alors même que Benda et Politzer, à la même époque, lancent des pamphlets violents contre le bergsonisme. ${ }^{14} \mathrm{Il}$ est assez caractéristique de lire le compte rendu de

\footnotetext{
${ }^{13}$ Jacques Herbrand, pourtant mathématicien salué par tous, rencontra les mêmes difficultés quand il chercha à introduire les idées hilbertiennes en France. Il n'eut ni le soutien des philosophes (Brunschvicg) ni celui des mathématiciens (Fréchet, Denjoy).

${ }^{14}$ Cf. [Benda 1912 (1926)], [Politzer 1947]. On pourrait penser que des articles
} 
la philosophie française des années trente donnée dans la Philosophical Review par Dominique Parodi [Parodi 1939]. On y trouve un éloge de la thèse de Cavaillès et des travaux de Lautman, présentés comme des disciples de Brunschvicg, et surtout comme des opposants au logicisme. Rougier n'y est mentionné que pour ses travaux sur le libéralisme, jugés «non dépourvus de biais partisans ».

Quand arrive la guerre, Rougier, en dépit de son activité effrénée, est un outsider dans le monde académique. Il est officiellement professeur à Besançon, mais il ne semble pas y avoir passé beaucoup de temps (outre son séjour au Caire, il enseigne en 1938 à l'institut des Etudes internationales de Genève, puis à la fondation Edouard Herriot à Lyon). Sa campagne pro-libérale a cependant plus de succès. Elle lui permet de se rapprocher de certains cercles du pouvoir et du patronat, et elle lui vaut, notamment par la collection qu'il dirige à la Librairie de Médicis et la fondation du Centre international d'Etudes pour la rénovation du libéralisme [Denord 2002], de connaître von Mises, Hayek, et Lionel Robbins, Jacques Rueff, et bien entendu Lippmann.

Le grand tournant de la vie de Rougier est 1940. Juste après la débâcle, il se retrouve à Vichy, où un peu toutes les élites françaises attendaient une affectation et l'issue des événements dramatiques de la débâcle. Il y semble très introduit dès le début : il a une réputation de technicien libéral, son collègue de Besançon le philosophe Jacques Chevalier, qui sera ministre de l'éducation nationale de Pétain, l'épaule. Il semble avoir participé d'entrée de jeu aux discussions du gouvernement Pétain, et en tous cas il s'en targue. Dans Mission secrète à Londres, il raconte avoir discuté de la fondation d'un parti unique avec Marcel Déat, et avoir réussi à convaincre le gouvernement Pétain de ne pas en créer un, se démarquant d'emblée des fascistes français (il ne cesse, dans

comme ceux que Rougier publia à ses débuts sur l'intuition [Rougier 1916a] et sur la mort des vérités nécessaires [Rougier 1913] fussent dirigés contre Bergson. Peut-être le sont-ils implicitement. Mais il ne dit mot de Bergson dans ces articles. Dans le Traité de la connaissance [Rougier 1955a, 204], on trouve bien une allusion à Bergson, et à son opposition entre la science qui connaît le relatif, et la métaphysique, qui connaît l'absolu. Rougier ici attaque l'idée bergsonienne d'une intuition qui irait au fond des choses, et soutient que le problème est vide de sens. Mais il cite ailleurs dans le même livre avec faveur une déclaration du bergsonien Le Roy sur la plasticité de l'esprit humain. (p. 424). Si l'on compare à nouveau le disciple de Poincaré avec son maître, on peut penser que les polémiques de Poincaré avec le philosophe bergsonien Le Roy sont bien plus vives (cf. [Poincaré 1905, ch. X, « La science est-elle artificielle?»]). Et Benda a bien vu les affinités des thèses mobilistes de Bergson et Le Roy avec celles de Rougier. D'une manière générale, le rationaliste Rougier en a plus contre le rationalisme mal compris que contre l'irrationalisme dont Bergson était le plus influent des représentants en France. 
Mission secrète, de dire tout le mal qu'il pense de Laval). Son influence lui permet d'aider Von Mises à passer aux USA. Surtout il est un proche de Weygand, et c'est par son intermédiaire qu'il reçoit son ordre de mission à Londres. L'histoire est à présent bien connue, même si la source la plus fiable n'est certainement pas les mémoires que Rougier lui-même lui a consacrés et qui sont, selon le mot de Paxton « devenus presque une industrie » [Paxton 1973], [Frank, R. 1992]. Toute l'affaire a été jugée, aussi bien par un certain nombre de ses acteurs que par les historiens, très sévèrement. Rougier semble avoir été un instrument de la politique d'attentisme de Vichy vis-à-vis des britanniques, mais s'être pris lui-même pour le dépositaire d'une mission historique bien plus importante. La mission échoue, notamment parce qu'au moment même où Rougier est à Londres, on apprend l'entrevue de Montoire, mais surtout sans doute parce que les Anglais n'y ont jamais cru, raison sans doute pour laquelle ils ont démenti après la guerre les accords. Rougier en tout cas ne semble pas avoir insisté, et il profite de l'obtention d'une bourse de la fondation Rockefeller, avec laquelle ses accointances libérales l'avaient mis en rapport, pour partir aux Etats-Unis, où il passe la majeure partie des années de guerre. Depuis le livre de Mehlman (2000), on connaît bien la période new-yorkaise de Rougier, son amitié avec Simone Weil, ses démêlés avec l'Ecole libre des Hautes Etudes et les milieux gaullistes, sa dénonciation non sans courage des «faux monnayeurs du patriotisme », et la manière dont il s'est mis, à partir de ces années à prendre le contre-pied de l'histoire pour défendre systématiquement la politique de Vichy en défendant la thèse de l'attentisme patriotique et du double jeu tactique du Maréchal.

Il ne démordra pas de sa défense de Pétain, et son énergie de se dément pas après-guerre. Après un court séjour à Montréal en 1945, il publie des plaidoyers pro domo pour justifier sa mission, et toute une série d'ouvrages tirés notamment de ses chroniques américaines, tels que La défaite des vainqueurs, La France en marbre blanc, la plupart édités à Genève $A$ l'enseigne du cheval ailé, un éditeur qui s'est apparemment spécialisé dans la publication d'ouvrages pétainistes. Dans ces livres perce l'amertume de Rougier et sa hargne, notamment contre de Gaulle. Il avait de bonnes raisons pour cela. Quand ce qui fut en partie le fruit de ses efforts d'avant guerre, la Société du Mont Pélerin fondée par Hayek et Von Mises, se réalisa, il en fut exclu, en raison de ses compromissions avec Vichy, et surtout de sa défense publique et constante de ce régime. Plus tard Maurice Allais, qui était lié à ces courants, rendit hommage à Rougier [Allais 1990], mais il était trop tard. Rougier aurait pu se taire et jouer profil bas. Nombre de collaborateurs le firent et pas- 
sèrent sous l'orage. Rougier n'était pas un collaborateur, mais son obstination à vouloir justifier Vichy pendant et après guerre fit qu'il apparut presque comme tel alors même que son implication avait été, comparée à d'autres, très temporaire. Avec un certain courage, mais aussi avec une grande témérité, il persiste et signe, et c'est sans doute cette apologie ouverte de Vichy qui lui vaut tous ses ennuis, qu'il semble rechercher. Son collègue Jacques Chevalier et bien d'autres qui avaient trempé bien plus que lui dans Vichy sont amnistiés. Mais lui est condamné par un tribunal d'épuration et suspendu d'enseignement entre 1948 et 1955, date où il retrouve un poste universitaire à Caen. On peut imaginer aisément quels furent ses sentiments quand il fut condamné. Pourtant, non seulement il ne cesse de travailler, mais il publie libelle sur libelle, sur l'épuration ou sur la politique, dans la ligne de ses critiques des institutions politiques françaises, comme La France jacobine, ou L'erreur de la démocratie française. Son anti-gaullisme et son pétainisme, ainsi que sa défense de la thèse du double jeu, le rapprochent des milieux ex-collaborateurs et même des fascistes qui, tels Bardèche, sont pourtant aux antipodes de ses idées libérales. On le retrouve dans un grand nombre de cercles d'extrême droite, des procès de l'après guerre à la guerre d'Algérie. ${ }^{15}$ Il ne justifie pas les actions de l'OAS, mais il est présent au procès de Bastien-Thiry [Rougier 1963b]. On connaît la suite.

Dans les années 70, Rougier connaîtra un regain de notoriété avec la redécouverte de son œuvre par Alain de Benoist et la «nouvelle droite ». Jean François Revel publie dans la collection «Libertés» son pamphlet Une faillite, La scolastique; Alain de Benoist son Celse contre les chrétiens. Son œuvre philosophique connait encore dans les années 50 et 60 un certain écho, avec le Traité de la connaissance (1955) et La métaphysique et langage (1960) qui n'apportent pas beaucoup par rapport à ses écrits d'avant guerre, mais qui sont estimés par les rares spécialistes. ${ }^{16}$ Mais ses dernières années sont surtout consacrées à la religion et à sa lutte anti-chrétienne. Il publie en 1969 son Génie de l'Occident, en 1972

${ }^{15}$ Il appartient à la société des amis de Brasillach, habite dans l'immeuble du Directeur des Ecrits de Paris, préface un ouvrage d'une rapatriée d'Algérie.

${ }^{16}$ [Granger 1956] rend compte du Traité de la connaissance en termes sinon élogieux, du moins sympathiques. Robert Blanché publie en 1967 dans l'Encyclopedia of philosophy de Paul Edwards un article (vol. VII pp. 217-18) fort élogieux sur Rougier, et au début des années 1960, il donne son «parcours intellectuel » dans le Tableau de la philosophie française de Denis Huisman [Blanché 1967]. Ensuite, les articles sur lui dans le domaine proprement philosophique se font très rares, sauf quand la Nouvelle Droite le redécouvre. La notice sur Rougier du Dictionnaire des philosophes des PUF ne comprend que quelques lignes et ne mentionne même pas ses écrits ni ses activités politiques. 
sa Genèse des dogmes chrétiens, et meurt, dans le même isolement que celui dans lequel il aura vécu, en 1982.

\section{Parallèle}

On peut essayer à présent de faire, à la manière de Plutarque, le parallèle éthico-intellectuel entre nos deux auteurs. Il est facile et simpliste de défendre au sujet de Rougier la thèse que j'ai appelée ci-dessus de l'implication stricte (1). C'est ce qui a conduit beaucoup de gens à rejeter en bloc l'œuvre de Rougier. L'antipathie profonde pour ses engagements pro-vichystes et d'extrême droite conduisit à dénigrer ses travaux de philosophie des sciences, voire même à considérer qu'il y a un lien intrinsèque entre la défense de thèse positivistes et les idées d'extrême droite. Bien qu'il y ait des raisons très nombreuses pour expliquer l'ignorance, le mépris, et dans le meilleur des cas le désintérêt pour les idées du positivisme logique en France, ${ }^{17}$ il est même probable que l'association de Rougier et du positivisme ait nui au second et que l'on ait affaire à un cas de culpabilité par association. Quand je me suis intéressé, durant mes années d'étudiant, vers 1970, au positivisme logique, j'ai souvent entendu cet argument, notamment de la part de mes camarades d'Ecole normale : puisque je lisais les positivistes et m'intéressais à la logique, je devais être nécessairement d'extrême droite (ou même catholique, comme me le dit une fois Althusser, dans ce que je ne perçus pas immédiatement comme un trait d'humour). Russell, Neurath, ou Carnap, qui étaient soit des socialistes soit des anarchistes, mais qui n'en avaient pas moins des idées proches de celles des positivistes, étaient mis dans le même sac que Rougier, et l'opprobre jetée sur ce dernier rejaillissait même sur la logique. ${ }^{18}$ Il ne venait d'ailleurs à personne l'idée de retourner l'argument et de soutenir que la logique était finalement une bonne chose, puisqu'un résistant comme Cavaillès s'y était consacré. L'autorité de résistant de Georges Canguilhem, qui détestait le positivisme logique et tout ce qui pouvait y ressembler, ne fut d'ailleurs pas pour peu dans ces attitudes, même si ce sont essentiellement les disciples d'Althusser qui développaient ces thèmes.

On a souvent été près de défendre une thèse proche de celle de l'implication stricte au sujet de Cavaillès, et on a vu encore la marque d'une

\footnotetext{
${ }^{17}$ Quelques indications dans [Engel 1996].

${ }^{18}$ Les travaux récents ont montré qu'il y avait en fait une droite et une gauche $\mathrm{du}$ Cercle de Vienne, comme le fait remarquer [Marion 2004] et [Marion, ce volume]. Rougier était manifestement plus proche de la première.
} 
nécessité spinoziste dans le lien entre sa croyance aux essences en mathématiques et son action de résistant. Ainsi on a soutenu que l'éducation protestante de Cavaillès, son engagement profond et durable dans le protestantisme, avaient des rapports étroits avec son engagement dans la résistance. Certes les protestants français ont la réputation d'être des hommes d'honneur et de vertu, mais leur foi religieuse ne les vaccina manifestement pas contre la collaboration avec Vichy, comme le cas de Couve de Murville [avant qu'il rejoigne les gaullistes] le montre, et les compromissions du pasteur Boegner et de la HSP avec le régime sont connues [Paxton 1973 (1977), 222]. Les résistants étaient de toutes sortes de confessions religieuses : catholique, juive, protestante, mais aussi agnostiques ou athées, comme les communistes. Encore moins le fait que Cavaillès ait été philosophe n'a joué de rôle, puisque un certain nombre de philosophes ont été des collaborateurs : André Rivaud, ${ }^{19}$ Jacques Chevalier, ${ }^{20}$ par exemple. Plus important est le fait que Cavaillès ait été mobilisé, et qu'il ait été un soldat. Comme je l'ai déjà signalé plus haut, la trajectoire de Marc Bloch ici est à rapprocher de celle de Cavaillès : même arrière-plan militaire, même désarroi devant la débâcle des troupes françaises, même patriotisme et désir d'action en réaction. Mais le fait d'avoir été mobilisé et d'avoir vécu la débâcle des troupes françaises ne semble pas non plus avoir été une condition nécessaire de l'entrée en résistance, puisque nombre de communistes, par exemple, peuvent avoir rempli cette condition et n'être entrés en résistance que plus tard. Comme l'ont montré les travaux récents, les trajectoires des résistants de la première heure sont extrêmement singulières (en dépit de la trame communiste, quoi de commun entre Marc Bloch, François Cuzin, Jacques Decour, George Politzer, Jean Guéhenno ou Jean Gosset?), bien que Cavaillès n'ait pas été lui-même une essence singulière en la matière.

On peut en dire autant, mutatis mutandis, de celle des collaborateurs et de ceux qui se sont trouvés associés au régime de Vichy. Hommes de la droite traditionnelle, authentiques fascistes, radicaux socialistes, exsocialistes et ex-communistes ou futurs socialistes après guerre (comme Mitterrand), futurs gaullistes, catholiques, protestants, canailles, crapules et individus égarés côtoyaient des athées, libéraux et positivistes comme Rougier. Le fait d'être libéral ne prédisposait pas plus à être vi-

\footnotetext{
${ }^{19}$ Sur André Rivaud, voir l'article féroce que lui consacre Marc Bloch dans l'Etrange défaite [Bloch 1946 (1990)].

${ }^{20}$ Jacques Chevalier, filleul de Pétain, bergsonien, professeur à Grenoble, ami de Rougier, fut l'un de ceux qui l'introduisirent au sein du gouvernement de Vichy. Il fut ministre de l'Education du Maréchal, condamné à la Libération, puis gracié. Il rétablit les « devoirs envers Dieu» dans l'Ecole Républicaine.
} 
chyssois que gaulliste, comme le montre le cas de Raymond Aron, qui participa aux travaux du colloque Lippmann, correspondait avec Rougier $^{21}$ et rejoint de Gaulle à Londres. Pourquoi Rougier se retrouva-t-il dans le panier de crabes de Vichy en 1940 ? Sans doute parce qu'il s'était rapproché, dans les années précédentes des milieux patronaux et qu'il était proche du gouvernement Reynaud et de Weygand. Il est vraisemblable qu'il appartenait plus au groupe de ceux que l'on a appelé les «techniciens » du régime de Vichy [Paxton 1973 (1977), 312]. Peut être parce qu'il espérait enfin jouer le rôle politique auquel il avait aspiré durant la décennie précédente, et qu'il joua brièvement avec sa mission. Peut être, comme il le laisse entendre, parce que face à la défaite, il pensait plus nécessaire d'agir rationnellement et en technicien qu'en se laissant porter par ses sentiments. Il présente lui-même son action comme guidée à la fois par des considérations stratégiques (continuer la guerre en Afrique du Nord et pour cela gagner du temps, comme le recommandait Weygand), des considérations humanitaires (interrompre le blocus britannique et permettre le passage d'aliments pour les enfants en zone occupée) et une forme de patriotisme rationnel (la carte attentiste était la meilleure selon lui). Mais il ne nous explique pas pourquoi, après l'échec de sa mission, son patriotisme lui parut s'exprimer mieux par un départ aux Etats Unis, où il protesta vigoureusement contre les faux patriotes qu'il rencontrait dans les milieux émigrés, qu'en restant en France auprès de ses compatriotes. Pourquoi, s'il pensait réellement que la politique de Pétain était la moins mauvaise en la circonstance, comme il n'a cessé de l'affirmer après la guerre, n'a-t-il pas cherché à rester à Vichy? Il y a, à mon avis deux raisons. La première est que sa mission ayant échoué, sa cote auprès des gens de Vichy n'était plus très bonne. La seconde est qu'il s'apprêtait à épouser Lucie Friedman, qu'il avait connue par l'intermédiaire de Schlick, qui était d'origine juive. L'assassinat du même Schlick en 1936 par un étudiant nazi qui lui reprochait de penser de manière « juive », la fuite de von Mises, lui-même juif, et de nombreux membres du Cercle de Vienne, aux USA, lui laissait assez voir de quelle nature était le régime hitlérien, et quel sort il réservait aux juifs. Il est douteux qu'il ait ignoré non seulement les lois anti-juives des nazis mais aussi celles que Vichy s'apprêtait à promulguer. ${ }^{22}$ Dès octobre 1940 il put apprendre la déportation de 6700 juifs allemands en France, les 3 et 4 octobre 1940 il put apprendre les premières lois antisémites de

\footnotetext{
${ }^{21}$ Mais il n'en dit mot dans ses Mémoires, ce qui indique encore l'ostracisme à l'encontre de Rougier de la part même de ceux qui sympathisèrent avec certains aspects de son action avant guerre.

${ }^{22}$ Voir l'adresse : http ://hypo.ge-dip.etat-ge.ch/www/cliotexte/html/camps.de.concentration.html.
} 
Vichy. ${ }^{23}$ Il aida von Mises à passer en France puis aux USA, profitant de ses relations avec les autorités. Quand il obtint lui-même une bourse Rockefeller, il en fit autant. Ce qui est plus étonnant est que, alors qu'il savait très bien quel sort pouvait être réservé par le gouvernement de Vichy aux juifs, cela ne lui ait posé aucun problème pour se mettre au service de Pétain et qu'il n'ait rien, dans ses livres après guerre, à redire à la politique de Pétain envers les juifs. ${ }^{24} \mathrm{Si}$ l'on peut bien voir quel enchaînement de circonstances a pu conduire Rougier à se mettre au service de Vichy en 1940, puis à justifier la politique de ce régime pendant son séjour new-yorkais et après la guerre, on comprend moins pourquoi un philosophe qui se voulait libéral au sens de Constant, de Tocqueville et des penseurs qu'il avait réunis au colloque Lippmann a pu avec tant de constance défendre un régime dont, c'est le moins que l'on puisse dire, les caractéristiques démocratiques et libérales ne sautaient pas aux yeux, même avant le retour de Laval en avril 1942. Que peut bien avoir de commun le libéralisme du Rougier et son anti-christianisme avec la révolution nationale et avec la nouvelle alliance du trône et de l'autel qu'elle voulait mettre en place? Sans doute, comme tant d'autres, Rougier a-t-il pensé que la situation exceptionnelle de 1940 requérait un régime autoritaire et d'exception, et sans doute ses polémiques d'après guerre prennent-elles quelques distances avec Vichy et Pétain, mais on ne voit pas pourquoi les critiques cinglantes qu'il y adresse à de Gaulle, à la thèse de l'homme providentiel et à la tentation bonapartiste constante chez les Français n'auraient pas dû aussi s'appliquer à un régime tel que celui de Vichy, même à ses débuts. Certes Rougier ne semble avoir défendu que la politique étrangère de ce régime, en vue de justifier son propre rôle au sein de celle-ci. Mais par son silence sur les autres aspects de cette politique, par son anti-gaullisme et ses connexions avec l'extrême droite française la plus dure après guerre, il a fortement compromis ce que sa position libérale pouvait avoir d'avant-gardiste avant guerre. Je ne crois donc pas à la thèse de l'implication stricte : rien ni dans les idées défendues par Cavaillès ni dans celles défendues par Rougier ne les prédisposait à suivre les parcours qui ont été les leurs. Il me semble tout

${ }^{23}$ Cf. [Paxton 1973 (1977), 224] : interdiction aux juifs d'enseigner dans la fonction publique, d'appartenir à des organismes élus, interdiction aux juifs du département de l'Allier, où se trouvait Vichy (!).

${ }^{24}$ On trouve certes ( par exemple La défaite des vainqueurs, [Rougier1947f, 56]) des déclarations sur la «guerre zoologique» d'Hitler chez Rougier, mais c'est une quasi citation de Renan. Il parle plus tard dans L'erreur de la démocratie française [Rougier 1963b], des camps et de la politique d'extermination des nazis, mais de manière très allusive et rapide. Il n'a en tous cas pas un mot sur la politique de Vichy dans ce domaine. 
aussi abusif de chercher à lire rétrospectivement dans ses thèses conventionnalistes en philosophie des sciences, dans son positivisme militant, ou même dans son libéralisme, les prémisses de son engagement auprès de Vichy et plus tard de l'extrême droite, que de lire dans le rationalisme idéaliste de Cavaillès et son protestantisme les prémisses de son engagement dans la résistance. ${ }^{25}$ De la même manière il me semble également vain de chercher à excuser les engagements politiques de Rougier par un argument du type «Oui, mais quel grand philosophe des sciences ». Cela ne me paraît pas meilleur que l'argument qui consisterait à jeter un doute sur les théories logiques de Frege sous prétexte qu'il était antisémite. En revanche, la thèse faible, mais par là même peu informative, des recoupements partiels (3), me paraît correcte. Certaines des thèses de Rougier permettent de toute évidence de comprendre certains de ses engagements, sans qu'on puisse soutenir qu'il y ait un lien nécessaire. Mais ce n'est pas cette question qui m'intéresse ici.

Rougier est un philosophe, il faut le juger comme tel, et juger ses thèses et ses arguments. Or lui-même, dans son œuvre, n'a cessé d'établir des liens entre le domaine de la rationalité théorique et le domaine de la rationalité pratique. Il a défendu une certaine interprétation historique du rôle des idées et de leurs applications pratiques. Et ce sont ses thèses théoriques au sujet des liens entre théorie et pratique qui m'intéressent. La valeur du travail de Rougier comme découvreur, son engagement pionnier dans des sujets qui n'avaient, dans la France des années 1920 et 1930 que très peu d'écho au sein des philosophes de sa génération, sont incontestables. Son isolement intellectuel aussi bien avant qu'après la guerre est largement dû au fait qu'à partir des années 1920 la majeure partie de la philosophie française a pris ses références dans la philosophie allemande, chez Hegel, Husserl et Heidegger. Les Autrichiens, et les Allemands qui, comme Carnap, ne défendaient pas les idées post-kantiennes, furent ignorés (et on peut dire que ce sort fut également en grande partie partagé par des kantiens comme Cassirer). Mais cela n'est pas propre à Rougier. Avant la guerre les idées du positivisme furent introduites par le Général Vouillemin et des mathématiciens comme Marcel Boll, mais elles demeurèrent très marginales. Des philosophes qui, comme Jules Vuillemin, écrivaient sur Russell dans les années 1960 furent eux aussi isolés. Mais l'isolement de Rougier et l'admiration

\footnotetext{
${ }^{25}$ On pourrait aussi mentionner les différences dans les éducations et les parcours universitaires. Cavaillès était normalien, parisien et bien installé au cœur de la voie royale de l'université française, Rougier provincial et marginal, ce qu'il accentuera par sa carrière à l'étranger. Mais on ne peut en tirer une quelconque généralisation : Brasillach aussi était normalien, comme Nizan.
} 
que nous pouvons avoir pour son engagement envers le positivisme dans les années 1930 ne doivent pas nous cacher les faiblesses de nombre de ses thèses philosophiques. Réservons-nous un jugement sur l'homme, mais évaluons d'abord ses idées.

Pourtant Rougier lui-même défend souvent la thèse de l'implication stricte entre les thèses philosophiques sur la théorie de la connaissance et les thèses sur la morale et la politique. Dès Les paralogismes du rationalisme Rougier parle des «conséquences » du rationalisme pour la morale et pour la politique. Il soutient que le rationalisme métaphysique, reposant sur une théorie des vérités nécessaires et a priori, sur l'identité de la raison en tout homme, conduit à la conception de l'égalité naturelle des hommes et des peuples, à la thèse de la souveraineté et la compétences universelle de la raison, à celle de la puissance de l'éducation sur les individus, et de l'autorité des lois pour les gouverner. Dans le domaine politique, il soutient que de ce même rationalisme « dérivent » l'égalité des droits politiques et la démocratie et au principe de la compétence de la majorité. Chez un logicien, cet usage des mots « conséquence» et « dérivation » est pour le moins curieux.

La même thèse est reprise quelques années plus tard dans La mystique démocratique, par exemple quand il déclare que le cartésianisme, suivant en cela Aristote et la scolastique ( $\mathrm{sic}$ ) est responsable des doctrines égalitaristes [Rougier 1929b, 87 et suiv.]. Rougier reconnaît pourtant bien qu'il ne peut y avoir d'implication directe du rationalisme cartésien (au sens de l'affirmation des idées innées) à la théorie de l'uniformité de la nature humaine puisque l'empirisme de Locke soutient, avec la thèse de la tabula rasa, la même idée sur des prémisses opposées. Il reproche à Locke de faire abstraction de l' « hérédité psychologique » (p. 94) ce qui peut laisser entendre qu'il soutient des doctrines héréditaires (mais il s'appuie sans doute plutôt sur Pareto ici).

L'argument me paraît à peu près aussi bon que celui qui faisait dire à Chomsky, dans Linguistique cartésienne, que l'empirisme était réactionnaire parce qu'il admettait l'influence de l'environnement sur la cognition et l'innéisme une doctrine de gauche parce qu'il admettait un noyau identique chez tous les hommes. Comme le reconnaît Rougier lui-même, on ne voit pas en quoi le fait d'être rationaliste, au sens où on admettrait des vérités nécessaires, a priori, et une forme d'intuition rationnelle, conduirait automatiquement à des doctrines égalitaires. En réalité, comme Rougier le rend clair, ce n'est pas le rationalisme lui-même qui conduit à l'égalitarisme qu'il condamne, mais la mystique rationaliste, ce qui est tout autre chose. Il définit une mystique comme « un ensemble de croyances qu'on ne saurait justifier en raison, ni en expérience, mais qui s'imposent 
par la voix de l'autorité, de l'habitude, du préjugé, de l'intérêt et plus particulièrement parce qu'elles expriment et sanctionnent les aspirations sentimentales et les tendances passionnelles d'un individu ou d'une collectivité. »Son exemple de mystique rationaliste est Rousseau. On ne peut nier qu'il y ait de telles mystiques. Mais Rougier écrit le plus souvent comme si toute forme de rationalisme souscrivant à une unité de la raison humaine ou à la thèse de l'existence de vérités nécessaires était automatiquement une mystique. Le concept de mystique est intéressant. Il fut sans doute suggéré à Rougier par Comte, Taine, Renan, et surtout par Pareto. Mais il ne fait pas l'objet d'une grande élaboration de sa part, contrairement à la théorie de l'irrationalité et des sophismes que l'on peut trouver dans la tradition de Bentham, de l'utilitarisme et chez Pareto lui-même avec sa théorie des « dérivations $»{ }^{26}$

Ce qu'il y a de plus problématique, aussi bien dans La mystique démocratique que dans les Paralogismes, me semble être la manière dont Rougier se livre à des équations saisissantes du type : rationalisme $=$ égalitarisme $=$ Révolution française $=$ judaïsme $=$ christianisme $=$ démocratie, ou tout au moins avec un signe d'implication fort à la place de $«=»$. Ce style d'empirisme spéculatif, consistant à brosser un tableau de la civilisation à larges traits, lui vient sans doute de Taine et de Renan, mais on ne peut pas dire qu'il le manie avec beaucoup de délicatesse. Sa méthode est bien souvent celle de ce que Bentham appelle, dans son Manuel des Sophismes politiques, celle des sweeping comparisons. ${ }^{27}$ Quand on lit certains raccourcis ${ }^{28}$ sur le judéo-christianisme, on

\footnotetext{
${ }^{26}$ cf. Pareto, Traité de Sociologie générale [Pareto 1916 (1968)] et [Bouvier (éd.) 1999]. Le concept de mystique est repris par Raymond Aron (sans citer Rougier, derechef), dans [Aron Raymond 1956].

${ }^{27}$ [Bentham 1824 (1996)]. Le plus joli exemple contemporain de sweeping comparison est celui de Bernard Henri Lévy, qui déclara un jour, en pleine mode des nouveaux philosophes, que puisque Lénine avait dit que le bolchevisme c'était les soviets et l'électricité, la pensée des Lumières et la raison éclairée devaient être, à l'instar du bolchevisme, des formes de dictature. On notera que la méthode des sweeping comparisons est un trait constant de la rhétorique française, notamment en matière politique, et que Rougier n'innove pas, pas plus que Lévy.

${ }^{28}$ p. ex. dans [Rougier 1929b, 12] et p. 164 : «l'utopisme philosophique du XVIII ${ }^{\mathrm{e}}$ siècle, conduisant au règne des tricoteuses et des sans culottes sous la Terreur ne sera qu'une sécularisation de l'apocalyptique d'Israël, tout comme le marxisme, établissant en Russie la dictature des ouvriers et des paysans, en est la transposition sur le terrain économique »; p. 176 : «Rien ne mesure mieux la distance qui sépare le génie hébreu du génie grec que la comparaison du Prométhée d'Eschyle avec le culte des anges et l'assomption d'Hénoch. Pour l'Hellène, la civilisation et la culture sont de merveilleux privilèges dont les dieux sont jaloux et leur jalousie qui cloue au Caucase le titan bienfaiteur de l'humanité »; pour Hénoch ce sont des œuvres diaboliques qui corrompent l'humanité et l'acheminent à sa perte; p. 180 sur le judaïsme de Marx : «Le judaïsme dit : Heureux les Bebionim, car le royaume de Dieu est à eux, et le
} 
se peut se poser quelques questions. Rougier a été accusé d'antisémitisme (Bounoure 1987) et son association avec la Nouvelle Droite n'y est pas pour peu. C'est sans doute une accusation fausse, mais il a certainement manifesté dans ses écrits une forme d'anti-judaïsme qui est très associée à son anti-christianisme.

Le style de Rougier rappelle quelquefois, dans ses écrits d'après guerre, celui que Albert Hirschmann a appelé « la rhétorique réactionnaire ». ${ }^{29}$ Mais son style réel, aussi bien en épistémologie qu'en philosophie politique, est plutôt celui du prophétisme. C'est rarement la bonne manière d'argumenter.

Que vaut la critique du rationalisme par Rougier? Dans Les paralogismes, il définit ainsi cette doctrine :

« Le rationalisme admet l'existence de vérités objectives, $a$ priori, inconditionnellement nécessaires, indépendantes de notre esprit et de la nature qui sont tout à la fois des lois de notre pensée et des lois de l'être. A ces vérités on donne le nom de vérités rationnelles ou de vérités éternelles. La faculté qui les appréhende, distincte de la perception sensible et de l'entendement empirique, est la raison. Cette faculté générique est une et indivisible. Elle est égale chez tous les hommes et leur appartient par essence et en tant que telle. » (p. 437)

On peut tout à fait souscrire à cette critique de la notion d'intuition rationnelle, qui rejoint celle que la tradition autrichienne adresse à la conception kantienne des vérités a priori tout comme à la tradition française cartésienne. Mais on a trop souvent l'impression que la démonstration de Rougier, ici, comme ailleurs, prouve trop. Au passage qui vient d'être cité, il s'empresse certes d'ajouter que « en dehors de ces deux propositions, le plus parfait désaccord règne entre les rationalistes », mais une thèse définie de manière aussi vague et massive ne peut pas servir de base à une histoire aussi vaste que celle qui est envisagée par Rougier.

La thèse qu'il oppose au rationalisme est, comme on l'a déjà dit, une forme de radicalisation du conventionnalisme de Poincaré que l'on peut appeler un empirisme pluraliste. Poincaré, comme l'ont remarqué

messianisme contemporain dit : L'union des prolétaires fera la paix du monde », etc.

${ }^{29}$ [Hirschmann 1989 (1991)]. Cf. notamment l'argument dit du « dangereux précédent » : «Le Principe de la Porte Ouverte commande de s'abstenir aujourd'hui d'agir avec justice de crainte de susciter l'espoir qu'on agira demain avec plus de justice encore - attente à laquelle on ne se sent pas le courage de répondre (...) Le Principe du Dangereux Précédent commande de s'abstenir d'accomplir aujourd'hui un acte que l'on sait être juste de crainte de manquer du courage nécessaire pour en faire autant demain dans un cas qui, pour différer fondamentalement de la situation présente, n'en présente pas moins avec elle une ressemblance superficielle. » 
nombre de ses interprètes, ne considérait pas que son conventionnalisme puisse impliquer une forme de relativisme : au contraire Poincaré est un réaliste. Mais Rougier considère que le conventionnalisme entraîne que toutes les structures de la raison, y compris celles de la logique et de la méthode scientifique, sont relatives et conventionnelles. Il suffit de lire l'article «L'évolution de la raison» dans les Dernières pensées, pour s'assurer que Poincaré était bien plus proche d'une forme de réalisme et qu'il croyait en une pérennité des cadres de la pensée rationnelle. ${ }^{30}$ Rougier lui-même ne défend certainement pas un empirisme radical, puisqu'il admet des propositions a priori. Celles-ci, cependant, sont simplement des conventions. L'empirisme de Rougier est pluraliste, parce qu'il soutient qu'il y autant de «mentalités » (on dirait aujourd'hui de «schèmes conceptuels ») qu'il y a de types de conventions dans une société donnée. Cela ressemble fort à une forme de relativisme. ${ }^{31} \mathrm{~A}$ la fin du Traité de la connaissance, Rougier entend défendre une théorie « évolutive» de la raison. ${ }^{32}$ On pourrait y voir une forme d'évolutionnisme. Certes Rougier cite Mach et le principe d'économie de pensée, mais il ne se livre à aucune spéculation darwinienne du type de celles auxquelles se livraient des auteurs comme Boltzmann par exemple. ${ }^{33}$ Comme l'a montré Benda (1950), il se situe plutôt dans la lignée de ces philosophes des sciences post-bergsoniens qui, à l'instar de Le Roy, voient dans la raison humaine une mobilité essentielle (j'y reviens ci-dessous).

En 1941, dans un article paru dans Phenomenology and Phenomenological Research, puis dans la Revue de métaphysique et de morale, repris quasi intégralement dans le Traité [Rougier 1941b], [Rougier 1955a, 423 et suiv.], Rougier défend la thèse selon laquelle il y a une pluralité de logiques rivales, sans qu'aucune n'ait de privilège autre que d'être au service des fins de l'action et de la connaissance empirique. La logique classique jouit des mêmes privilèges que la géométrie euclidienne pour la perception, mais pas plus. L'article de 1941 fit l'objet, dans le Journal of Symbolic Logic, d'une critique assez dure de Julius Kraft [Kraft 1942]. Ce dernier demande : comment peut-on dériver la logique, qui est supposée consister en une infinité de vérités, de conventions qui ne sont elles

\footnotetext{
${ }^{30}$ Cf. [Poincaré 1913b] et [Zahar 2001] sur le « réalisme structural » de Poincaré.

${ }^{31}$ Notons que Rougier ne fut pas le seul à interpréter Poincaré en ce sens. L'école positiviste polonaise, et notamment K. Ajdukiewicz, défendit un « conventionnalisme radical » de ce type, qui impliquait une forme de relativisme.

${ }^{32}$ Cf. notamment p. 427 .

${ }^{33} \mathrm{La}$ seule spéculation est in [Rougier 1955a, 428] qui fait référence au principe d'économie de pensée de Mach qui « a son origine dans les conditions de notre vie biologique ». Rougier, comme l'a montré [Marion 2004] était pourtant anti-réductionniste et anti-physicaliste. Il serait donc un peu étonnant qu'il envisage réellement une conception naturaliste de la raison humaine.
} 
mêmes ni vraies ni fausses? Kraft reproche à Rougier de ne considérer que le calcul propositionnel et de négliger celui des prédicats, alors que ce dernier donne des arguments pour isoler la logique du premier ordre des autres logiques. On notera aussi que, à l'exception de quelques allusions dans le Traité, Rougier ne fait pas une grande place aux considérations métathéoriques de type gödelien.

Que valent les arguments de Rougier? Ils me paraissent valoir ce que vaut la thèse de la dérivation de la logique à partir de conventions et la division analytique/ synthétique de Carnap. A aucun moment Rougier ne prend en compte les critiques de Quine à Carnap, publiées en 1936 dans «Truth by Convention ». ${ }^{34}$ Il ne tient aucun compte des critiques de « Two Dogmas of Empiricism » (1951), qu'il ne semble pas connaître. ${ }^{35}$ Sa culture logico-mathématique semble s'être arrêtée au début des années 30 .

Ce que dit Rougier de la pluralité des logiques a un lien étroit, sinon implique, ce qu'il dit des cadres généraux de la pensée rationnelle. Comme on l'a déjà noté, son premier article publié, en 1917, porte sur la mort des vérités nécessaires, et il étend cette idée à l'ensemble des propriétés de la raison. Mais il fait ici une confusion entre la raison comme faculté d'une part et la raison comme méthode et ensemble de normes d'autre part. Le fait que la première, en tant que capacité immuable à saisir par l'intuition des vérités nécessaires, soit douteuse, n'implique en rien que la seconde le soit. Et le fait que les vérités scientifiques changent, tout comme les théories et les cadres de la science, ne signifie en rien que la raison comme ensemble de critères et de normes de la connaissance varie également.

Rougier ici comme ailleurs semble bien de son temps, et pas aussi original qu'il le semble. Il est assez curieux que, tout occupé à attaquer le rationalisme sous ses diverses incarnations, il ne consacre, le plus souvent, pas beaucoup de développements à l'irrationalisme dans ses écrits. Il dénonce certes les mystiques rationalistes, mais très peu les mystiques tout court. Et à la différence, par exemple, de Russell, qui polémiqua violemment contre Bergson, on ne trouve pas chez lui de critique de Bergson. Or Bergson était, de tous les philosophes français, le plus influent. Julien Benda, mais aussi George Politzer, parmi ses contemporains, s'attaquèrent à Bergson au nom du rationalisme. Mais Rougier ne

\footnotetext{
${ }^{34}$ Mathieu Marion me fait remarquer que le texte est cité dans la bibliographie du Traité. Mais il ne semble pas avoir été utilisé.

${ }^{35}$ Quine a donné une critique fameuse de Lévy-Bruhl et de la notion de mentalité primitive, que Rougier acceptait. Il est clair que Rougier ignorait ces développements de l'empirisme logique après guerre.
} 
le fait pas. Il semble avoir apprécié, chez Bergson, les dénonciations des paralogismes et des pseudo-problèmes [Rougier 1955a, 296], mais il ne l'associe, et pour cause, jamais à sa hargne contre le rationalisme.

Julien Benda a parfaitement identifié le paralogisme commis par ceux qui confondent la variabilité des vérités avec la variabilité des cadres de la pensée rationnelle. Il dénonce chez ses contemporains le «mobilisme » et la thèse du caractère «plastique » de la raison, qu'il identifie chez de nombreux auteurs, en l'occurrence Bergson, Brunschvicg, Le Roy, Bachelard et Rougier. Citant ce dernier il écrit, dans De quelques constantes de l'esprit humain :

« La confusion que nous marquons ici se montre, ou plutôt se cache, dans le procès que les dynamistes intentent au « rationalisme classique » parce qu'il aurait cru «à l'existence de vérités universelles et nécessaires et par conséquent immuables » ou même lorsqu'il dénoncent, parmi les «paralogismes du rationalisme» sa croyance à des « vérités scientifiques nécessaires » et son refus de « reconnaître ce qu'elles ont de contingent $»[$ Benda écrit en note : Rougier op cit, p. 441, et « le même réquisitoire est constant chez Brunschvicg »]. Il y a là une équivoque, dont on se demande si ceux qui la pratiquent le font inconsciemment ou par calcul. Le rationalisme «classique » en tant qu'il est scientifique ne croit nullement à l'existence de vérités immuables, si l'on entend par vérités des états de la pensée estimés adéquats à la réalité; il sait fort bien que ces états sont sujets à laisser la place à d'autres plus qualifiés et il est en cela parfaitement «dynamique ». Ce qu'il tient pour immuable, c'est le critère en raison duquel l'esprit appelle ses assertions des vérités. » [Benda 1950, 119]

La mutabilité des vérités scientifiques est une chose, celle des normes de la rationalité en est une autre, et il n'y a aucune raison de passer de l'une à l'autre. Pour le faire, il faut un argument montrant que les cadres de la pensée rationnelle changent à travers les siècles et les cultures. Mais Rougier ne le donne pas. Il se contente de le présupposer. Quand il décrit les diverses «mentalités»- comme la mentalité «scolastique » en supposant qu'elles ont chacune leur métaphysique, Rougier est beaucoup moins continuiste que Comte avec sa théorie des trois états. Il radicalise là aussi Lévy-Bruhl et la notion de mentalité qu'il trouve chez ce dernier. ${ }^{36}$ Si l'on veut un bon argument contre ce mobilisme, on

\footnotetext{
${ }^{36} \mathrm{~A}$ certains moments, sa description fait presque penser au relativisme structuraliste d'un Foucault. Cf. [Rougier 1955a, 272].
} 
le trouvera là aussi, et non sans noter l'ironie de la chose, chez Poincaré. Dans 《l'évolution des lois » [Poincaré 1913b] ce dernier soutient que la science ne peut pas faire l'économie d'un métaprincipe général selon lequel la science doit tenir ses lois fondamentales comme immuables, même si, dans le passé, les savants reconnaissent avoir formulé des conjectures fausses. Comme l'a parfaitement vu Benda, Rougier est un parfait disciple de la conception française des années 20-30, qui a son origine chez Bergson, et que l'on retrouve de Brunschvicg à Bachelard, du mobilisme de la raison. En ce sens il est disciple non de Poincaré, mais plutôt de Le Roy, l'apôtre d'un conventionnalisme mâtiné de bergsonisme.

De même les arguments de Rougier dans La métaphysique et le langage (1960), pour montrer que les catégories de pensée sont relatives aux catégories de langue, qui reproduisent ses déclarations antérieures quand il soutenait en 1920 que la langue grecque prédispose à la métaphysique parce que l'article défini peut être placé devant un adjectif, hypostasiant les notions (to agathon), ou que les langues sémitiques qui n'ont pas de verbe être sont moins métaphysiques, sont bien peu convaincants, et encore moins ses appels à une réforme du langage et de la syntaxe qui produirait, à l'entendre, une réforme automatique de la pensée. Les vues de Rougier sur les relations entre langage, ontologie, et signification, nous paraissent bien naïves aujourd'hui, car nous avons lu Quine et Davidson sur l'indétermination de la traduction et parce que nous ne pensons plus qu'on puisse décalquer aussi aisément les catégories de pensée à partir des catégories de langue. Mais même Benveniste, qui écrivait à la même époque sur ces sujets, n'était pas aussi simpliste que Rougier, qui semble en être resté, sur ce point comme sur d'autres, aux idées du Carnap de « Le dépassement de la métaphysique par l'analyse logique du langage » (1931).

Passons, pour finir, aux vues de Rougier sur les valeurs morales, qui ne peuvent qu'avoir une certaine pertinence pour notre problème. Là aussi, sa position officielle est strictement positiviste. Comme Durkheim et Poincaré, et comme la tradition positiviste, il ne voit aucun passage possible du fait à la valeur ou au droit, et il sépare radicalement jugements de faits scientifiques et jugement de valeur. ${ }^{37} \mathrm{Il}$ ne défend pas aussi nettement que Carnap l'émotivisme en éthique (influence de Schlick?), mais il est parfaitement clair qu'il est un anti-réaliste. La question classique, quand on défend ce type de conception est : comment éviter le relativisme moral? Il semble souvent considérer que la morale se ré-

\footnotetext{
${ }^{37}$ [Rougier 1963b, 124] : la science ne concerne que les jugements assertoriques, pas les jugements de valeur. Il ne peut y avoir de morale scientifique. La science n'a rien à voir avec l'éthique » et [Rougier1955a, 417].
} 
duit à la science des mœurs ou à la sociologie (se rappeler la encore Lévy-Bruhl, Morale théorique et science des mœurs) et qu'une bonne ingénierie sociale pourra remplacer les trémolos éthiques. Il n'y a aucune implication nécessaire entre ces vues et telle action politique, bien qu'il y en ait d'évidentes entre ces vues et le libéralisme politique. Carnap était un socialiste, et Neurath un homme d'extrême gauche. Comme on l'a déjà noté, nombre des libéraux associés aux entreprises de Rougier avant guerre furent ensuite gaullistes.

Quand il présente les résultats de ses actions politiques, Rougier adopte là aussi souvent le langage de l'ingénieur social et de l'efficacité. Il lui paraît aussi important, pour défendre la France, de s'occuper du ravitaillement des enfants et de l'état de l'Empire que de chercher à faire alliance avec les Anglais. Sa mission fut certainement inspirée par ce souci de la Realpolitik. Mais n'est pas Realpolitiker qui veut, et l'équipée à Londres le montre. On se serait attendu à ce que Rougier, dans ses écrits en justification ex post facto durant la guerre et après, se réclame, pour justifier sa position et son action pro-vichyste, d'un argument d'efficacité. Il le fait en partie, mais en même temps il se recommande du patriotisme et de la morale, notamment quand il s'oppose aux gaullistes new-yorkais, ce qui est pour le moins paradoxal en comparaison de sa position officielle. Kant refusait la notion de fortune morale, et Bernard Williams a rejeté ses arguments. Russell disait que l'entrée en politique des philosophes était une bonne chose quand leur action est réussie. Il est difficile en effet de ne pas juger Rougier au regard des résultats de son action, qui ont été, il faut bien le dire, ratés. On ne peut s'empêcher de penser qu'il aurait mieux valu pour lui qu'il eût une conception un peu moins instrumentaliste de la raison dans le domaine éthique, et plus réaliste ou cognitiviste s'agissant des valeurs morales. Ici son positivisme, sa conception purement subjectiviste de la morale (et passablement pro domo dans ses polémiques d'après guerre), lui jouent des tours. La seule justification qu'il trouve des valeurs morales est qu'elles sont celles d'une population à un moment donné, ce qui revient, contre son révolutionnarisme libéral, à prôner le conservatisme (cela peut expliquer son adhésion à Vichy). Le rôle de la politique est réduit par Rougier à celui d'une pure technique. Du moment qu'on a les élites, tout est supposé se mettre en place (même en admettant cela, encore aurait-il fallu qu'il nous dise comment on recrute les-dites élites, or on ne trouve chez le Professeur Rougier aucune réflexion décente sur l'université). Là encore, le contraste avec Cavaillès, mais surtout avec Benda est édifiant, dans la mesure où, à la différence de Cavaillès, Benda a théorisé son rationalisme éthique. Benda demeura en France en 1940 ; il se réfugia à Carcassonne, 
après que les autorités de Vichy lui aient confisqué sa bibliothèque. ${ }^{38} \mathrm{Il}$ ne renonça jamais à sa conception éternitaire de la raison. Le jugement de Rougier sur Benda est contenu au détour d'une page de la Mystique démocratique :

« La grande question semble bien plutôt résider dans la sélection d'une élite spirituelle qui reprendrait, dans la société de demain, l'honneur et le rang qui lui sont dus, et dans la formation d'un public de gens ayant suffisamment de loisirs pour acquérir la politesse des mœurs et le goût des plaisirs de l'esprit. Elle consiste dans la possibilité de dériver, hors du circuit fermé de l'économie capitaliste, une part de l'énorme circulation des richesses, en vue de la faire servir à des fins qui n'aient pas nécessairement par elles-mêmes une valeur directement marchande : le grand art, la science spéculative, la culture désintéressée. Alors seulement les clercs, suffisamment indépendants pour se dispenser de trahir, assistés par un public de vrais connaisseurs, se détourneront de l'idole informe, Belphégor, pour revenir au culte délaissé d'Athéna. » [Rougier 1929b, 79]

Les élites ou les clercs, là est la question. Mais on ne peut pas dire que les élites de Vichy se soient tellement bien comportées, comparées aux clercs. Mais peu importe. Julien Benda ne cesse, dans toute son oeuvre, de dénoncer «la fin de l'éternel » et la confusion des idéaux théoriques avec les objectifs pratiques, historiques, et pragmatiques. On ne saurait trouver, chez le juif républicain et chez le catholique lyonnais devenu antichrétien, des positions plus opposées, et on ne saurait trouver, chez le spinoziste Cavaillès et le libéral-positiviste Rougier, des chemins de vie plus divergents.

L'incontestable mérite de Rougier réside dans son courage intellectuel et sa curiosité insatiable, qui lui donnèrent le souci d'aller chercher les idées dans la tradition positiviste à laquelle il se rattachait, et l'audace de défendre, dans une France toute tournée vers les idées allemandes, des principes viennois. Mais au-delà, le lamentable fiasco de ses activités politiques après guerre, son association avec l'extrême droite, et son souci permanent d'avoir malgré tout raison, expliquent en grande partie son destin.

Une chose me paraît en tous cas sûre. Le moins que l'on puisse dire est que chez Rougier, la croyance au caractère changeant, plastique et dynamique de la raison, le sens aigu de la contingence de la vérité vont

\footnotetext{
${ }^{38}$ Voir l'excellent article de [Malkassian 2002].
} 
de pair avec une grande confiance en lui-même et en l'autorité de ses propres idées et principes d'action, et tout autant dans ses engagements politiques effectifs. Il y a là quelque chose qui tranche avec le faillibilisme manifesté par de nombreux positivistes. ${ }^{39}$ Dans une page des Accords Pétain Churchill, Rougier cite Louis XIV :

«C'est d'un petit esprit que de vouloir ne s'être jamais trompé, et ceux qui ont assez de mérite pour réussir le plus souvent trouvent quelque magnanimité à reconnaître leurs propres fautes. »[Rougier 1945b, 287]

Il ne semble jamais lui avoir traversé l'esprit que cela pourrait s'appliquer à lui-même. ${ }^{40}$

\footnotetext{
${ }^{39}$ Bien qu'il faille noter que le plus faillibiliste en théorie - Popper - ne l'est guère dans ses attitudes.

${ }^{40} \mathrm{Je}$ remercie de leurs encouragements et de leurs commentaires Serge Audier, Mathieu Marion, Kevin Mulligan, Jean-Claude Pont et Antonia Soulez.
} 